\section{Nonlocal Finite Element Analysis OF STRAIN-Softening Solids}

By Zdeněk P. Bažant ${ }^{1}$ and Ta-Peng Chang ${ }^{2}$

Abstract: A two-dimensional finite element formulation for imbricate nonlocal strain-softening continuum is presented and numerically demonstrated. The only difference from the usual, local finite element codes is that certain finite elements are imbricated, i.e., they regularly overlap while skipping the intermediate mesh nodes. The element imbrication is characterized by generating proper integer matrices that give the numbers of the nodes for each finite element and the numbers of the imbricate elements overlapping each local element. The number of unknown displacements remains the same as for a local finite element code, while the number of finite elements approximately doubles. Numerical results show that stable two-dimensional strain-softening zones of multiple-element width can be obtained, and that the solution exhibits proper convergence as the mesh is refined. The convergence is demonstrated for the load-displacement diagrams, for the strain profiles across the strain-softening band, and for the total energy dissipated by cracking. It is also shown that the local formulations exhibit incorrect convergence; they converge to solutions for which the energy dissipation dwe to failure is zero, which is physically unacceptable. Stability problems due to strain-softening are avoided by making the loading steps so small that no two mutually nonoverlapping elements may enter the strain-softening regime within the same load step.

\section{INTRODUCTION}

Distributed damage, such as distributed cracking, can be macroscopically described as strain-softening, a situation in which the matrix of tangential elastic moduli ceases to be positive definite. Beginning with Hadamard in 1903, many investigators have demonstrated various difficulties which this phenomenon introduces in structural analysis $(6,8,9,28,29,33)$. These difficulties, which are caused by material instability of the strain-localization type, lead to physically unreasonable results for which energy dissipation in the material is confined to zones of zero volume (surfaces, lines or points) (8). If such complete strain localization is prevented by assuming smooth macroscopic strain distributions, problems are encountered with uniqueness (28-30).

The difficulties disappear $(9,22,23)$, however, when the material is described as a nonlocal continuum, in which the macroscopic (homogenized) stress at a point depends not only on the macroscopic (homogenized) strain at the same point but also on the entire strain field in a certain neighborhood of the point. The classical nonlocal continuum formulation $(19,22-25)$ was shown to be appropriate for elastic statistically heterogeneous materials. However, the classical formulation was found unworkable for strain-softening, and a new special type of nonlocal con-

'Prof. of Civ. Engrg. and Dir., Ctr. for Concrete and Geomaterials, Northwestern Univ., Evanston, IL 60201

${ }^{2}$ Struct. Engrg. Specialist, Sargent and Lundy Engrs., Chicago, IL 60603; formerly Grad. Research Asst., Northwestern Univ., Evanston, IL 60201

Note.-Discussion open until June 1, 1987. To extend the closing date one month, a written request must be filed with the ASCE Manager of Journals. The manuscript for this paper was submitted for review and possible publication on September 30, 1985. This paper is part of the Joumal of Engineering Mechanics, Vol. 113, No. 1, January, 1987. CASCE, ISSN 0733-9399/87/0001-0089/\$01.00. Paper No. 21182. tinuum, called the imbricate continuum, was formulated and its applicability demonstrated $(5,9,15)$. This continuum, which represents the limit of a system of imbricated (regularly overlapping) elements of a fixed size, mathematically differs from the classical nonlocal continuum models in that the field operator is self-adjoint. This is because the gradient averaging operator is applied not only to the strains, but also to the stresses obtained from the strains. In contrast to the classical local continuum models, one obtains a symmetric structural stiffness matrix if the material stiffness matrix is symmetric. As another difference from the classical nonlocal formulations, it was found necessary to overlay the nonlocal continuum with a local one in order to assure stability of discrete finite element approximations $(9,12,15)$.

Finite element formulations based on the idea of imbricate nonlocal continuum have been formulated and shown to converge as the finite element mesh is refined $(9,15)$. The convergence of the explicit time-step algorithm for dynamic problems was found to be quadratic (15). For the special case of a strain-softening local continuum, the finite element solution was shown to converge to the exact solution of a strain-softening wave propagation problem for a one-dimensional local continuum (8). These solutions, however, have so far been confined to one-dimensional problems of linear, cylindrical, and spherical geometries $(4,9,15)$. Although a multidimensional imbricate finite element formulation was proposed (5), it has not yet been numerically implemented. This is the objective of the present study.

It should be mentioned that various alternative models have been recently proposed for the description of progressive distributed cracking; see, e.g. Refs. 6, 26-27, 31, 32. However, examination of the relative merits of various models as well as experimental verification is beyond the scope of this paper and can be found elsewhere; e.g. Ref. 6.

\section{Review of Imbricate Nonlocal Continuum}

The imbricate nonlocal continuum is described by the following relations $(5,9)$ :

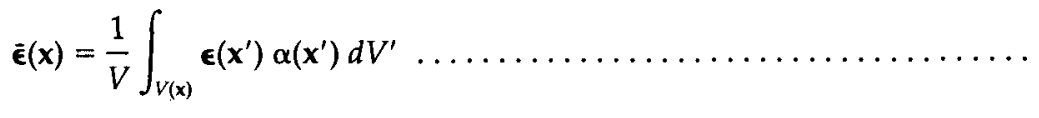

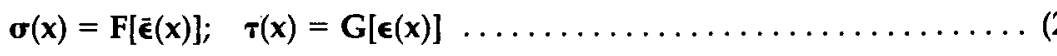

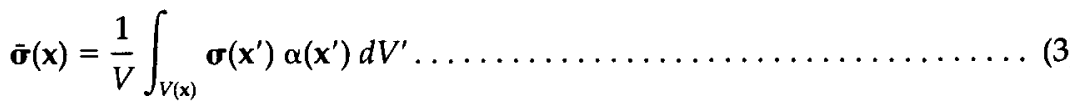

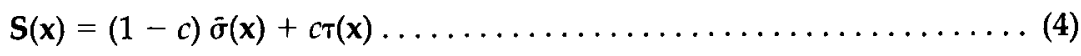

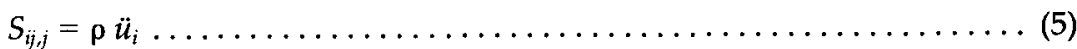

in which $\epsilon$ is the strain tensor, $\bar{\epsilon}$ is the mean strain tensor, $\tau$ is the local stress, $\boldsymbol{\sigma}$ is the broad range stress, $\mathbf{F}$ and $\mathbf{G}$ are the constitutive operators, of which only $\mathbf{F}$ may exhibit strain-softening, $\overline{\boldsymbol{\sigma}}$ is the mean broadrange stress, $\mathbf{S}$ is the total stress with cartesian components $S_{i j}(i, j=$ $1,2,3), V(\mathbf{x})$ is the characteristic volume (of volume $V$ ) centered at point 
$x, \alpha(x)$ is the empirical weighting function (normally $\alpha=1$ ), $\rho=$ mass density, $u_{i}=$ cartesian displacement components, and $c$ is an empirical participation factor for the local behavior.

There are two essential differences from the classical nonlocal continuum theory: (a) The averaging operator appears not only in Eq. 1 defining the mean strain, but again in Eq. 3 defining the stress that is substituted into the continuum equation of motion (Eq. 5); and (b) a local continuum must be imagined to be overlaid over (or coupled in series with) the nonlocal continuum, i.e., coefficient $c$ cannot be zero. For $c=$ 0 the continuum would be unstable, which is manifested by an unresisted periodic strain field of zero energy $(9,12)$. Theoretically, any value $c>0$ suffices for stability, but for very small values, such as $c=0.01$, excessive noise is obtained in numerical simulation; in practice, $c=0.1$ usually suffices to suppress the noise.

The representative volume $V$ may be imagined in two dimensions as a circle of diameter $\ell$ or a square of side $\ell$ where $\ell$ is a characteristic length of the medium. This characteristic length may be considered to be approximately the same as the size of the fracture process zone which can be determined experimentally on the basis of fracture tests $(1,3,7,13)$. The most indicative are the tests of geometrically similar fracture specimens of different sizes. The characteristic length $\ell$ appears to be approximately related to the maximum size of the inhomogeneities within the material, such as the maximum aggregate size in concrete or the maximum grain size in rocks or sea ice.

The foregoing field equations represent the continuum limit of an imbricated (regularly overlapping) finite element system as the mesh size is refined to zero $(5,9)$ and, conversely, the discrete approximation of the foregoing field equations is represented by such an imbricated finite element system. We restrict our attention to two-dimensional finite element analysis using rectangular meshes. In this case, the side of the nonlocal finite elements cannot be smaller than the given characteristic length $\ell$. This means that if the mesh size is smaller than $\ell$, the finite element must span over several meshes and be connected only to the mesh nodes at the element boundary but not to the mesh nodes within the element area. This leads to an imbricate arrangement of nonlocal finite elements; see Fig. 1 which shows the imagined cross section of the element system and a view of the plane of finite elements in which the square imbricate elements are slightly rotated out of alignment so as to permit their visual distinction. Although the element system is pictured by means of several layers of imbricated elements, the imbrication is fictitious. There is actually no third dimension and, mathematically, all elements are laid out in the same plane.

In a regular square mesh of imbricated square elements representing a continuum of thickness 1 in the third dimension, each point other than the nodes is overlapped by one local square element of thickness $c$ and by $n^{2}$ imbricated square elements; $n$ is the number of meshes spanned by the imbricate element of size $\ell$ and $n=\ell / h$. The mesh size $h$ should be chosen so that $n$ is an integer. Since the thicknesses of all overlapping elements must add up to 1 , each of the imbricate elements has the thickness $(1-c) / n^{2}$.

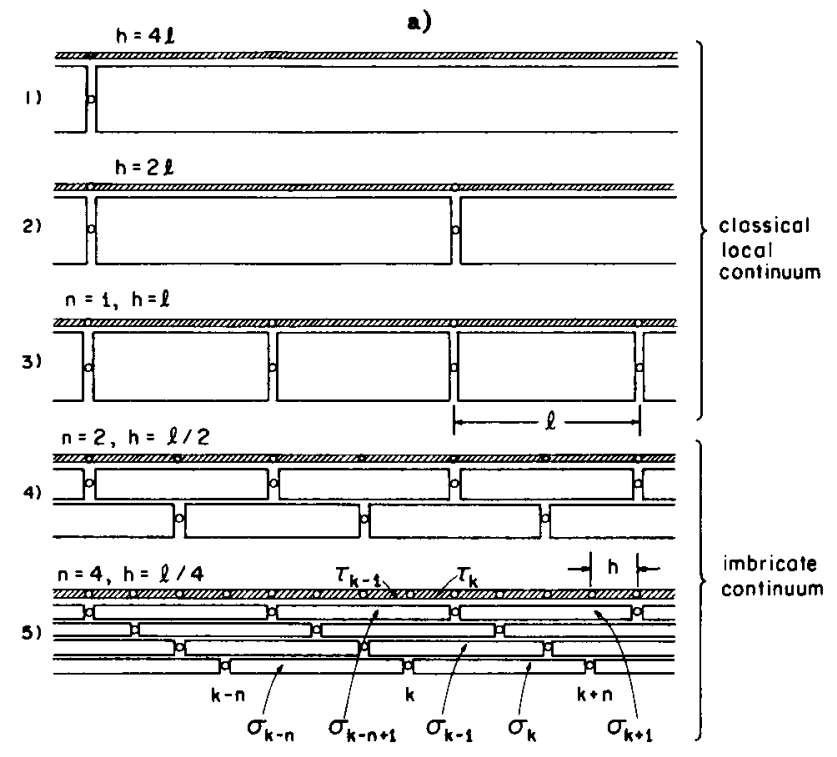

b)

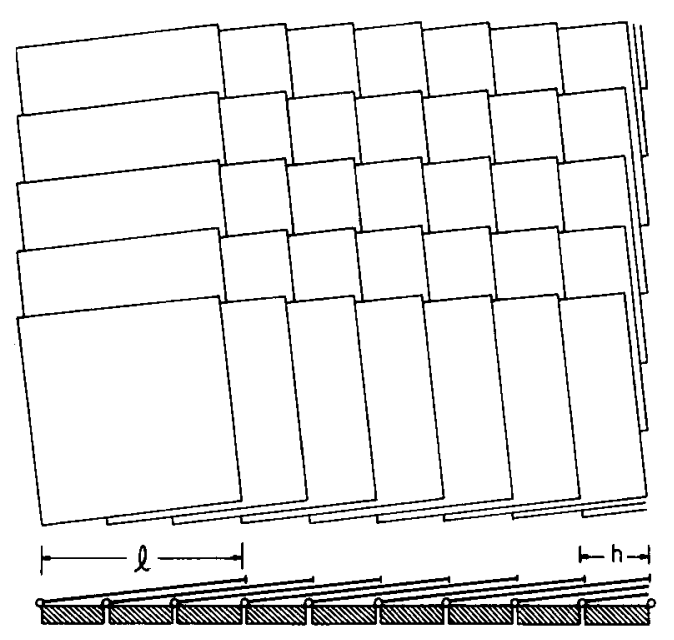

FIG. 1.-(a) Expanded Cross Section of Imbricate Finite Element Meshes at Increasing Mesh Refinements (Actual Thickness Is Zero); (b) Plan View of Imbricate Elements Slightly Rotated Out of Alignment for Purpose of Illustration

The boundaries are most easily handled by laying out a regular imbricate element mesh imagining at first the imbricate elements to protrude outside the boundary. Then the protruding parts of the elements are imagined to be chopped away and the element nodes outside the boundary are moved onto the boundary and made to coincide with ap- 
propriate nodes of other elements lying at this boundary. Thus, many of the boundary elements are smaller than the interior elements and do not meet the restriction that the element size should not be smaller than $\ell$; however, this is inevitable and acceptable since a nonlocal continuum obviously involves a boundary layer of thickness $\ell$ which requires special treatment and has in fact different governing field equations in the continuum limit. Imagining the protruding parts of the imbricate elements to be chopped off is a convenient way to model the boundary layer, and is simpler than determining first the field equations for the boundary layer and then attempting to formulate their discrete approximation

This handling of the boundary is of course intended only for rectangular four-node elements. The use of higher-order imbricate elements would introduce additional questions beyond the scope of this paper. Note, however, that the nonlocal imbricate aspect of the mesh is important only in the regions of strain-localization with strain-softening, i.e. at and near the fracture process zone, while elsewhere regular (nonimbricated) elements can be used with little effect on the results.

Any available finite element code based on the usual, local continuum concept can easily be generalized for nonlocal analysis by introducing the element imbrication. All that needs to be changed is: (1) To generate a nodal connectivity matrix, i.e., the integer matrix which specifies the nodal numbers for each finite element number; and (2) to generate another integer matrix which specifies for each square of the mesh the numbers of all elements that overlap that mesh. The former integer matrix is used in the usual manner to determine how the stiffness coefficients of the imbricate nonlocal elements and the local elements should be assembled into the structural stiffness matrix, while the latter integer matrix is used to determine how the stresses from all overlapping elements (i.e., the imbricate and local elements) should be added to obtain the total stress in the imbricate nonlocal continuum.

The stiffness matrix of the structure is assembled in the usual manner from the stiffness matrices of the imbricate nonlocal elements and the local elements; it may be expressed as:

$\mathbf{K}=\sum_{i=1}^{1} \frac{1-c}{n^{2}} \mathbf{k}_{i}^{i m}+\sum_{j=1}^{J} c \mathbf{k}_{j}^{\ell o c}$

in which $I$ and $J$ are the numbers of all imbricate and all local finite elements, and $\mathbf{k}_{i}^{i m}$ and $\mathbf{k}_{j}^{\ell \circ c}$ are their stiffness matrices written in the global numbering system. Note that the total number of finite elements is not substantially increased by element imbrication; for domains much larger than the size of the imbricate elements, the number of imbricate elements is approximately the same as the number of the local elements. The element imbrication, however, increases the band width of the structural stiffness matrix. For a rectangular domain and a rectangular mesh with $n_{x}$ and $n_{y}$ elements in the $x$-and $y$-directions, the minimum half-band width is

$n_{b}=2\left[n\left(n_{1}+2\right)+1\right] ; \quad n_{1}=\min \left(n_{x}, n_{y}\right)$

\section{Simple Constitutive Relation with Strain-Softening}

We assume the material to behave in a time-independent manner and for the purpose of numerical studies, we consider a rather simple constitutive relation with strain-softening which was introduced in Ref. 13, although more sophisticated constitutive relations $(6,14)$ could be also used.

We assume that the material is linearly elastic, and characterized by Young's elastic modulus and Poisson's ratio $v$, until the maximum principal tensile stress $\sigma_{1}$ reaches the tensile strength limit $f_{t}^{\prime}$. After the strength limit is reached, we assume a progressive formation of microcracks such that a unique stress-strain relation exists and the uniaxial tensile stressstrain diagram in the strain-softening portion is given by a straight line of negative slope $E_{t}$ (the strain-softening modulus). After the tensile stress drops to zero, the material has no resistance to further tensile extension. If contraction (negative strain increment) occurs, the material response is always elastic. For the sake of simplicity we assume that all the microcracks, assumed to be continuously distributed (smeared), have the same direction, which remains constant even if the direction of the maximum principal stress rotates. The direction of the microcracks for each integration point of each finite element is fixed once and for all at the moment when the tensile strength $f_{i}^{\prime}$ is reached. This direction is denoted as $y^{\prime}$ and the normal direction as $x^{\prime}$. After the start of progressive microcracking, i.e., during the strain-softening, the material is described by the incremental constitutive relation

$\left\{\begin{array}{l}\Delta \epsilon_{x}^{\prime} \\ \Delta \epsilon_{y}^{\prime}\end{array}\right\}=\left[\begin{array}{cc}E_{t}^{-1} & -\nu E^{-1} \\ -\nu E^{-1} & E^{-1}\end{array}\right]\left\{\begin{array}{l}\Delta \sigma_{x}^{\prime} \\ \Delta \sigma_{y}^{\prime}\end{array}\right\}$

in which $\Delta$ denotes increments, and $\epsilon_{x}^{\prime}, \epsilon_{y}^{\prime}, \sigma_{x}^{\prime}$ and $\sigma_{y}^{\prime}$ are the normal components of the strains and stresses in coordinates $x^{\prime}$ and $y^{\prime}$. The compliance matrix in Eq. 7 is constant throughout the strain-softening range, and $E_{t}<0$. Since the principal stress directions may rotate, but the axes $x^{\prime}$ and $y^{\prime}$ must be kept fixed at each point of the material, Eq. 7 needs to be generalized to allow for shear stresses $\tau_{x y}^{\prime}$ to be transmitted across the microcracks. The capability to transmit shear stresses due to crack roughness and aggregate interlock is a well-known property of concrete. To model it, Eq. 7 may be inverted and the shear stress expression then superimposed:

$\left\{\begin{array}{l}\Delta \boldsymbol{\sigma}_{x}^{\prime} \\ \Delta \boldsymbol{\sigma}_{y}^{\prime} \\ \Delta \boldsymbol{\sigma}_{x y}^{\prime}\end{array}\right\}=\left[\begin{array}{ccc}E_{t}^{\prime} & \boldsymbol{\nu} E_{t}^{\prime} & 0 \\ \nu E_{t}^{\prime} & E+\nu^{2} E_{t}^{\prime} & 0 \\ 0 & 0 & \beta G\end{array}\right]\left\{\begin{array}{c}\Delta \boldsymbol{\epsilon}_{\dot{x}}^{\prime} \\ \Delta \boldsymbol{\epsilon}_{\dot{y}}^{\prime} \\ \Delta \boldsymbol{\gamma}_{x y}^{\prime}\end{array}\right\} \ldots \ldots \ldots \ldots \ldots \ldots \ldots \ldots \ldots \ldots \ldots$

in which $E_{t}^{\prime}=E_{t} E /\left(E-\nu^{2} E_{t}\right) ; \gamma_{x y}^{\prime}=$ shear angle in coordinates $x^{\prime}$ and $y^{\prime} ;$ and $\beta$ is an empirical shear retention factor, as previously introduced by Schnobrich and others (34). In element coordinates, the incremental stressstrain relation in the strain-softening range is given as $\Delta \sigma=C_{t} \Delta \epsilon$, in which $\boldsymbol{\sigma}=\left(\sigma_{x}, \sigma_{y}, \tau_{x y}\right)^{T}, \epsilon=\left(\epsilon_{x}, \epsilon_{y}, \gamma_{x y}\right)^{T}, \mathbf{C}_{t}=\mathbf{T}^{T} \mathbf{C}^{\prime} \mathbf{T}$, where $\mathbf{C}^{\prime}$ is the stiffness matrix from Eq. 8 and $\mathrm{T}$ is a $3 \times 3$ transformation matrix the elements of which are $T_{11}=T_{22}=\cos ^{2} \theta, T_{12}=T_{21}=\sin ^{2} \theta, T_{32}=$ $-T_{31}=2 T_{13}=-2 T_{23}=\sin 2 \theta, T_{33}=\cos 2 \theta$, in which $\theta$ is the inclination 
angle of the axis $x^{\prime}$ with regard to the element coordinate axis $x$.

After the maximum total tensile stress at any point drops to zero, the tangential elastic modulus is considered as zero, $E_{t}=0$. For unloading defined as a reversal of sign of $\Delta \epsilon_{x}^{\prime}$, the initial elastic stiffness matrix is used. The same matrix is also used for reloading until $\epsilon_{x}^{\prime}$ exceeds its previous maximum, the record of which must be kept for each integration point throughout the computation.

The most important consequence of cracking is energy dissipation. The energy dissipated per small volume $\Delta V$ of the material during the $r$ th loading step may be calculated as

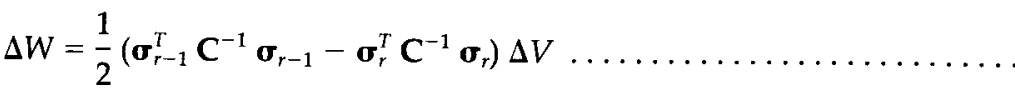

in which $\boldsymbol{\sigma}_{r-1}$ and $\boldsymbol{\sigma}_{r}$ are the column matrices of stress components at the beginning and the end of the $r$ th loading step.

The foregoing constitutive relation with strain-softening can be applied only for the imbricate nónlocal elements, i.e., the elements the size of which is at least $\ell$. For reasons of stability and convergence, the local elements, which can be refined to zero with the mesh, must not exhibit any strain-softening. They can exhibit elastic behavior or plastic behavior without softening (i.e., hardening-plastic or ideal-plastic). Thus, if the strain-softening law terminates with zero stress, the normal component

a) Local Formulation

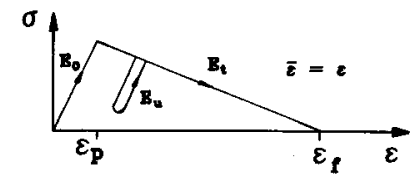

b) Imbricate Formulation
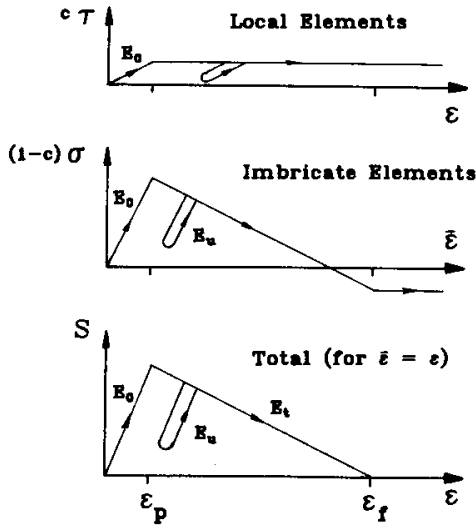

FIG. 2-Uniaxial Stress-Strain Diagrams Considered for Local and Imbricate Nonlocal Elements $\left(E_{u}=E_{0}=E\right)$ of total stress $\mathbf{S}$ in the direction normal to cracking does not actually become zero when strain-softening is terminated in the imbricate nonlocal elements. To make the total stress component vanish at a sufficiently large strain, one must introduce for the imbricate nonlocal elements a constitutive law with over-softening; as shown in Fig. 2. The strain-softening portion dips below the horizontal axis until, at strain $\epsilon_{f}$. it reaches a constant negative value which exactly cancels the positive yield stress in the local elements, with yielding assumed to begin at the same strain.

When the mesh size is equal to or larger than $\ell$, the imbricate and local elements are identical and can be fused into a single element. The strain-softening constitutive law shown in Fig. 2(a) can then be used directly.

\section{Numerical Studies}

As an example, consider the rectangular panel shown in Fig. 3. The panel has a sliding support at the base and free boundaries on both sides. On the top side, the nodes are free to move horizontally while their vertical displacements are increased monotonically in small steps $\Delta u$ which are the same for all nodes. If the material were perfectly uniform, the strain and stress fields would be also uniform in the hardening range. To obtain strain concentrations that trigger strain localization and ultimately lead to fracture, we assume that in small regions around the midlength points of the specimen sides the tensile strength is $3 \%$ lower than in the rest of the panel, in which the strength is uniformly distributed. The small difference in strength suffices to nucleate a zone of strainlocalization propagating from the specimen sides inward.

The material properties are defined (nondimensionally) as follows: Tensile strength $f_{t}^{\prime}=1.0, E_{0}=100, E_{t}=-10$, except for the weaker

\section{a) Mesh I}

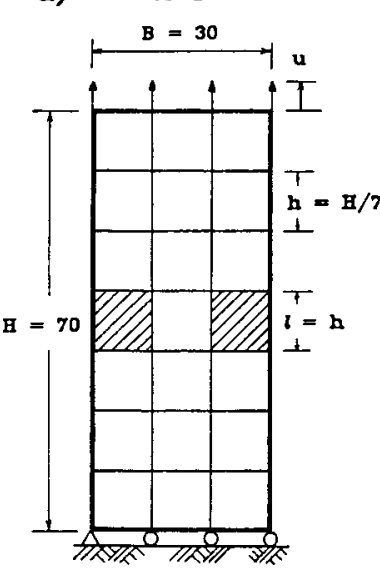

b) Mesh II proscribed

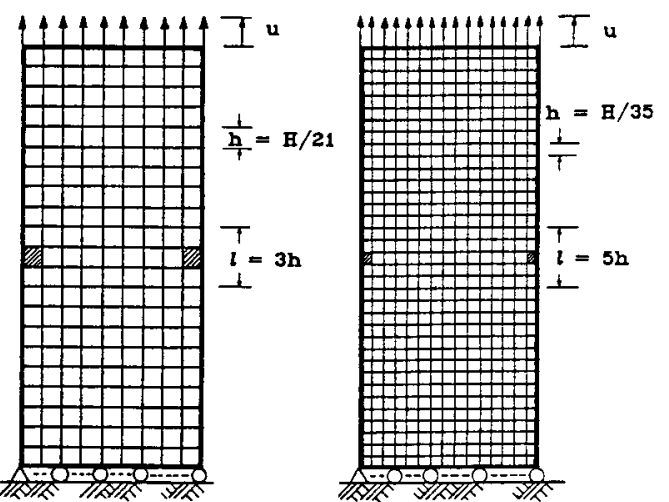

FIG. 3.-Mesh Refinement for Rectangular Panel with Weaker Elements at Sides (Shaded) 
element for which $f_{t}^{\prime}=0.97$ and $E_{t}$ is chosen so that the declining portion of the stress-strain diagram terminates at the same point $s$ as it does for the stronger elements. Also, $c=0.1$ and Poisson's ratio $\nu=0.2$.

Convergence with mesh refinement is a crucial question with strainsoftening models. To study convergence, we select the three square meshes shown in Fig. 3, with mesh sizes in the ratio 1:1/3:1/5. In each mesh only one element on each panel side, shaded in Fig. 3 , is assumed to have the lower strength limit. The numbers of local (square) elements for the three meshes $(1,2$, and 3 ) are 21,189 , and 525, respectively, and the total numbers of the imbricate elements are 21, 253, and 741, respectively. The numbers of the nodal points for the three meshes are 32,220 , and 576 . The number of imbricate elements which overlap each square of the mesh is 1,9 , and 25 for meshes 1,2 , and 3, respectively. The panel, its loading, and the meshes are symmetric with regard to the symmetry axes of the rectangle.

For comparison, solutions with the medium and finest meshes (II and III) have been also obtained uşing the usual, local finite elements.

Assuming the weaker regions at mid-sides of the panel to be smaller than the elements of mesh III, the weak region is modeled for each mesh by a single weaker element at each side of the panel. If we assumed the weaker regions to have the size of the elements of mesh $I$, then the weak regions for meshes II and III would consist of more than one element at each side, and the weak regions for all three meshes would then have identical dimensions. This would cause the convergence for mesh refinement to be even better than for the present example, and so this case would be a less demanding test for our theory.

Due to the bilinear form of the stress-strain relation, it seems most effective to use for the loading steps the tangential stiffness algorithm, except for those steps in which the state of the finite element is getting over the peak of the stress-strain relation or beyond the terminal point of strain-softening. All finite elements are four-node quadrilaterals (squares) with four numerical integration points per element. However, the material properties are not evaluated separately for the individual integration points; rather, the incremental material properties are assumed to be uniform for the entire element and are determined from the strain components at that numerical integration point for which the maximum principal strain is maximum among all integration points. When the maximum principal strains are the same in two integration points, which happens in our problem due to symmetry, all strain components from these two points are then averaged. The computational algorithm in each loading step can be briefly described as follows.

1. DO loop on iterations of the current loading step.

2. DO loop over all imbricate elements.

3. Based on the principal strain at the beginning of the load step, the maximum of the previous strains at that point and the loading-unloading-reloading condition from the previous load step (for the first iteration) or from the previous iteration (for the second and further iterations), choose the proper tangent material stiffness matrix for the element, i.e., Eq. 7 or Eq. 8 [or Eq. 8 with $E_{t}=0$, for the horizontal segment of $\sigma(\bar{\epsilon})$ diagram in Fig. $2(b)]$.
4. For the second and further iterations: If the final stress state from the previous iteration is not on the stress-strain diagram (which happens if the material state passed through the peak stress point during the previous iteration), calculate the initial stress $\Delta \sigma^{\prime \prime}$ that needs to be added in order to put the final stress state on the stress-strain diagram (the vertical stress drop in Fig. 4).

5. Calculate the element stiffness matrix and the residual nodal force equivalent to $\Delta \sigma^{\prime \prime}$, and assemble them into the structural stiffness matrix and the load vector in the usual manner.

6. Last element?-if not, go to step 2 .

7. Check how many elements have passed through the peak stress point during this load step, and print this number, $N_{p}$.

8. DO loop over all local elements.

9. Same as steps $2-6$, except that after reaching the plastic limit in Fig. 2(b) the perfectly plastic tangential stiffness matrix for the local elements is used.

10. Solve the linear equation system, and determine the increments of nodal displacements, stresses and strains.

11. Check the given convergence criterion, e.g., the condition that the sum of the absolute values of the latest changes of all residual nodal forces, divided by the sum of the absolute total values of all nodal forces must be less than a certain small positive number chosen by experience, such as 0.0001 . If not, return to step 1 and repeat the iterations. If the convergence is slow, reduce the loading step $\Delta u$. Then increment the values of displacements, stresses and strains and start the next loading step.

The most difficult problem is to detect all possible instabilities for equilibrium path bifurcations due to strain-softening. Stability criteria for strainsoftening $(2,6)$ are needed when more than one finite element enters the strain-softening regime during the same loading step. Thus, the stability checks may be avoided for the local formulation if the loading step is so small than no more than one finite element (in the present case, one element per quadrant, due to the symmetry of the rectangular domain) is permitted to enter the strain-softening regime during the loading step.

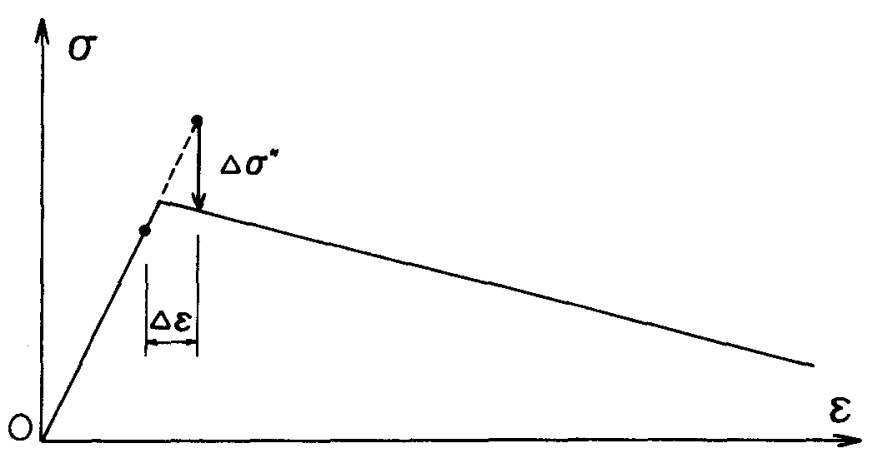

FIG. 4.-Initial Stress for Step $\Delta \epsilon$ that Crosses Stress Peak 
With the coarse mesh I, in which only local finite elements are used, the foregoing condition of no more than one element per quadrant to enter softening is satisfied by using boundary node displacement increments $\Delta u=0.005 u_{0}$ where $u_{0}$, is the displacement corresponding to a uniform deformation over the rectangle with the strain equal to that for the peak stress. For the finer meshes II and III, for which nonlocal imbricate elements are used, the foregoing condition requires an impracticably small loading step and appears unnecessarily stringent. Numerical experience showed that loading steps $\Delta u=0.0005 u_{0}$ for the medium mesh (II), and $\Delta u=0.0001 u_{0}$ for the finest mesh (III), are sufficient (i.e., give results that do not differ appreciably from those for smaller loading steps), even though more than one element may enter softening during one loading step. But this does not appear to cause a problem because the overlapping of the imbricated elements tends to stabilize the softening domain. It appears necessary, though, to make the loading step so small that no two nonoverlapping elements may enter the strain-softening regime within the same loading step.

For those loading steps in which no finite element passes through the peak point of the stress-strain diagrams-a case which occurs at the be-
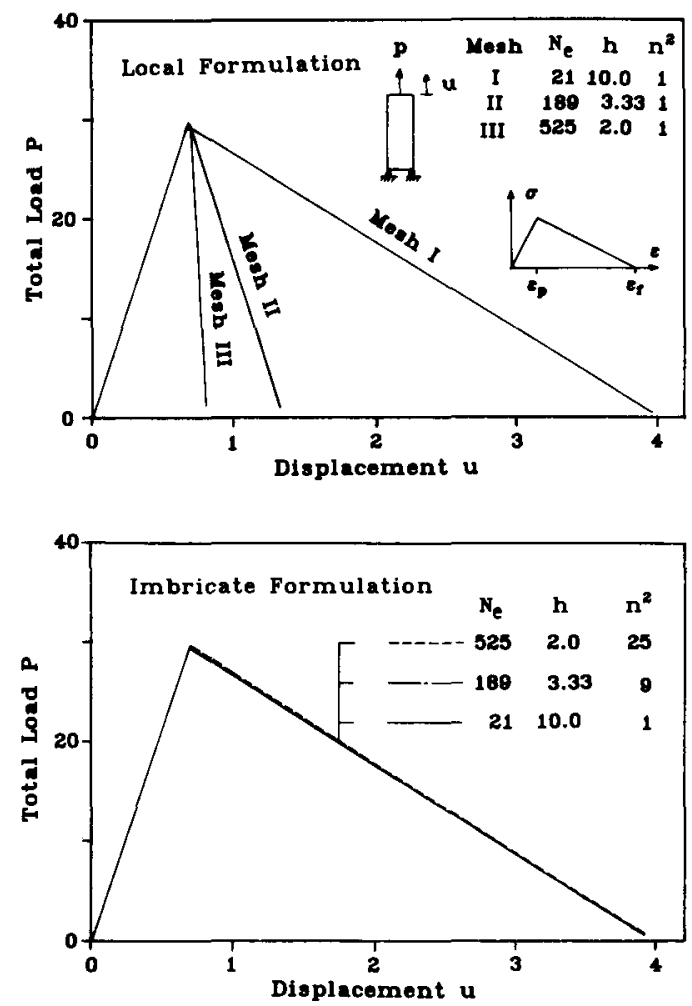

FIG. 5.-Diagrams of Load Versus Displacement at Top Boundary Obtained for Various Meshes with Local and Imbricate Formulations ginning of the analysis and again after the initiation of the complete crack band-one can of course use much larger loading steps. Then the use of the tangential stiffness matrix, as opposed to other methods such as the initial stress method, brings about considerable savings of computer time.

Comparisons between the local and imbricate finite element solutions are shown in Figs. 5, 6, and 7 in which the response diagrams of load versus boundary displacement, the profiles of vertical normal strain across the strain-softening zone, and the values of energy $W$ dissipated (consumed) due to strain-softening (cracking) are given. It is seen that incorrect convergence occurs when the local continuum is used. The softening slopes change greatly (Fig. 5) as the mesh with local elements is refined. In fact, for meshes finer than III, the softening slope in Fig. 5 (top) would revert to a positive slope, in which case the system becomes unstable under displacement control.

The strain profiles across the cracking band in local analysis are seen
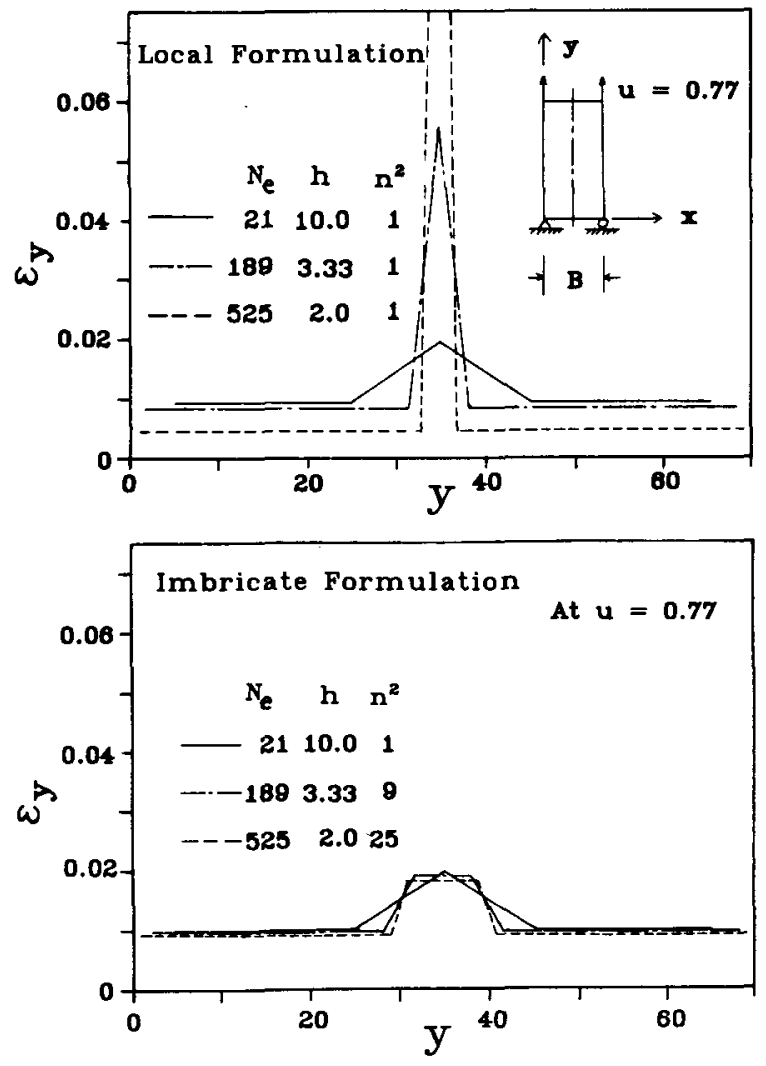

FIG. 6.-Profiles of Vertical Normal Strain at Vertical Line of Symmetry across Strain-Sottening Band Obtained for Various Finite Element Meshes with Local and Imbricate Formulations 

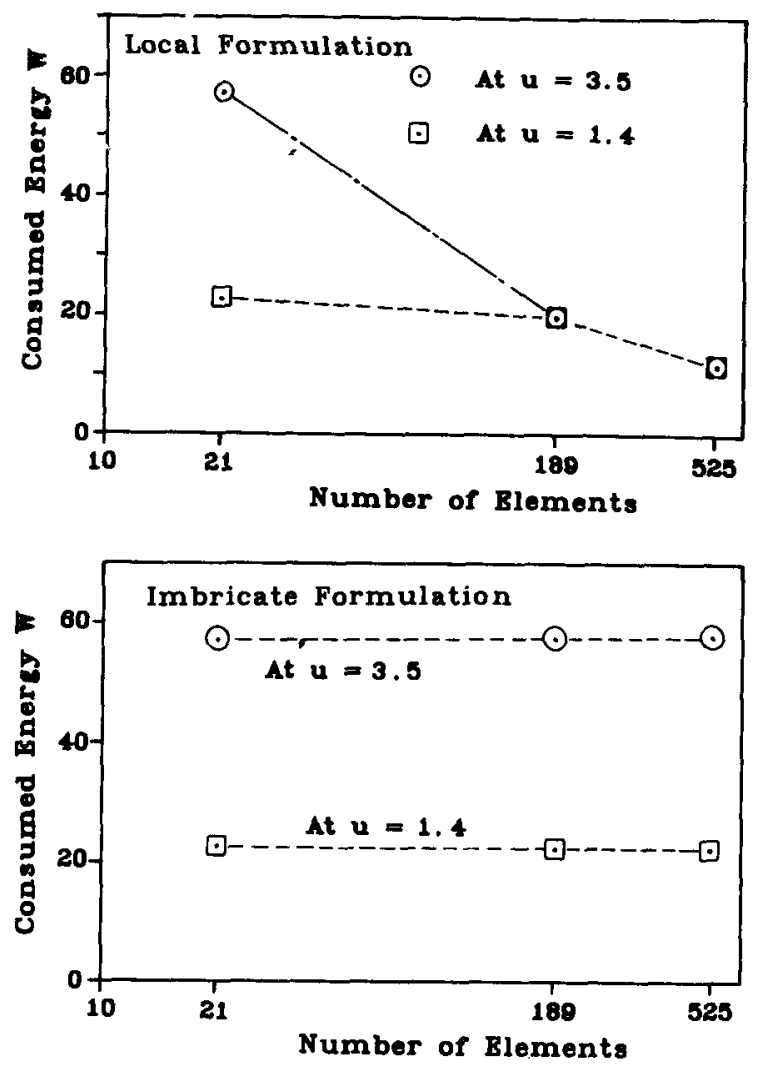

FIG. 7.-Diagrams of Total Energy Dissipated Due to Strain-Softening Versus Number of Elements for Local and Imbricate Formulations

to localize (Fig. 6, top) into progressively sharper spikes, apparently converging to a Dirac delta function. Since the dissipation of energy per unit volume of material is finite, and the strain-softening zone within the local continuum model appears to converge to a line, the total energy dissipation must converge to zero. This is confirmed in Fig. 7 (top) where, for the local finite elements, the dissipated energy $W$ decreases with the increasing number of finite elements. This also agrees with what is seen from Fig. 5 (top), in which the area under the load-displacement diagram represents the total energy dissipated by cracking; this area is also seen to converge to zero.

From the foregoing results (Figs. 5-7), it is clear that the local finite element model converges to a physically unreasonable solution, for which the failure is obtained at zero energy dissipation. This conclusion is similar to that made previously for the exact solution of wave propagation in a one-dimensional strain-softening bar (28), and agrees with the spurious mesh sensitivity of local finite element solutions $(6,10,13,28)$.

For the imbricate elements, by contrast, good convergence is apparent from Figs. 5, 6 (bottom) and 7. This again agrees with what has been previously demonstrated for one-dimensional problems $(9,15)$. The extent of the strain-softening zone (cracking zone) is seen in Fig. 8, in which the dots represent the centers of the elements that have passed through the peak point of the stress-strain diagram. For meshes II and III, these dots represent the centers of the imbricate elements. For the finest mesh we see that the strain-softening zone can have the width of several finite elements while remaining stable. It is also seen that the strain-softening zone in imbricate elements may have non-softening enclaves; note the uncracked (blank) element surrounded by strain-softening elements in Fig. 8, mesh III. Also note that the front of strainsoftening may be either pointed (mesh II) or blunt (mesh III).

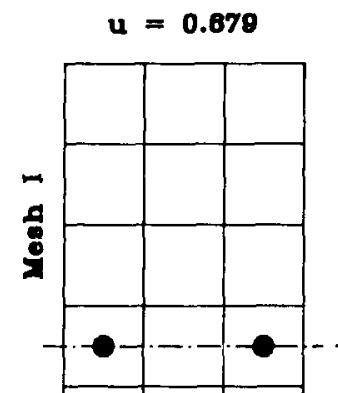

$\mathbf{u}=0.694$
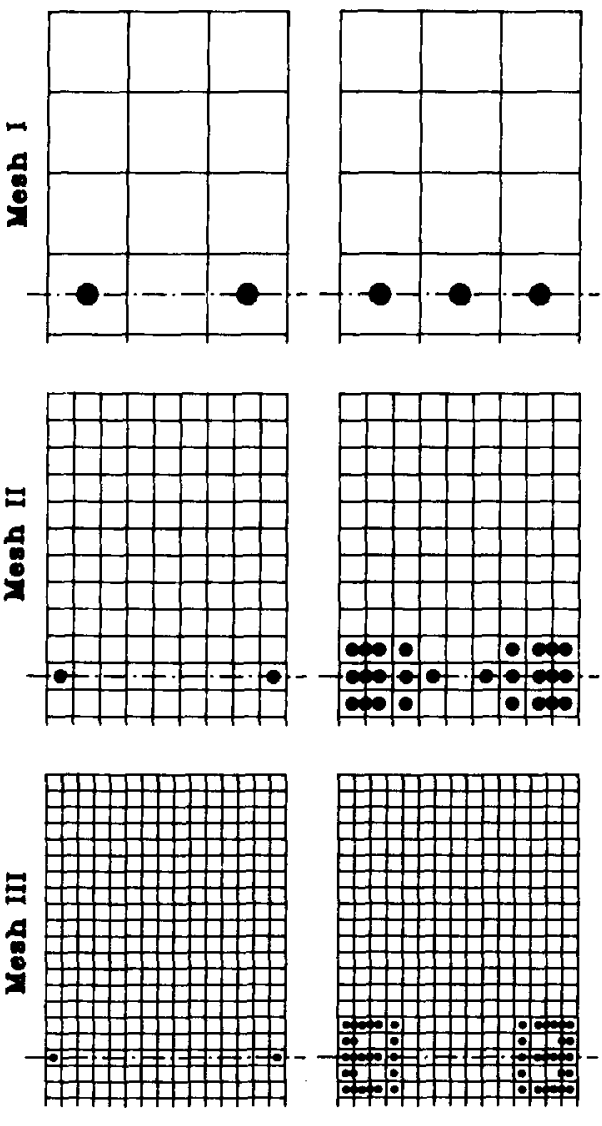

: conter of cracked elements $\mathbf{u}=0.7$
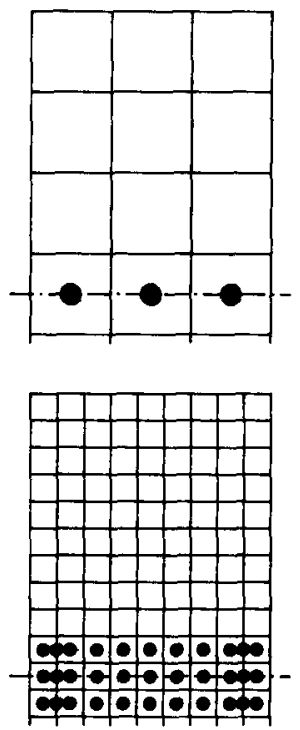

FIG. 8.-Centers (Marked by Dots) of Local (Mesh I) or Imbricate (Meshes II and III) Finite Elements that Have Entered Strain-Softening at Various Stages of Loading 


\section{Conclusions}

1. A finite element program based on imbricate nonlocal continuum can be easily obtained by modifying a local finite element program. The principal modification required is the generation of the integer matrices giving the numbers of all nodes that correspond to each element number, and the numbers of all imbricate elements that overlap each local element. Compared to a local finite element program with the same mesh, the number of finite elements is approximately doubled while the number of nodal displacements remains the same. For mesh sizes not smaller than the characteristic length of the continuum, the nonlocal imbricate finite element formulation is identical to the local finite element formulation.

2. Generalization of the present formulation to irregular meshes is possible, however the mesh must be regular in the region where the elements are imbricated, i.e., where the element size is less than $\ell$. The regular elements may be squares, but they could also be regular triangles or hexagons.

3. The two-dimensional imbricate finite element program is capable of modeling stable strain-softening zones of multiple-element width.

4. Numerical studies confirm that the solution of strain-softening problems converges as the mesh is refined. The convergence is demonstrated for the load-displacement diagrams, for the strain profiles across the strain-softening band, and for the total energy dissipated by cracking (strain-softening)

5. The local finite element formulation exhibits incorrect convergence with mesh refinement. It converges to a physically meaningless and unacceptable solution for which the area of the strain-softening zone and the energy dissipated due to failure are zero and the strains within this zone are infinite.

6. As the numerical demonstration of convergence confirms, stability problems due to strain-softening are avoided by imposing, for the local finite element formulation, the condition that no more than one finite element may enter the strain-softening regime in a single loading step. For the nonlocal formulation with a finer mesh, this condition may apparently be relaxed due to the stabilizing nature of the imbricate elements; however, the loading steps may not be larger than those for the local finite element program with the mesh size equal to the characteristic length of the continuum. Also, the load step should be so small that no two finite elements that do not overlap each other may enter the strain-softening regime within the same loading step.

\section{ACKNOWLEDGMENT}

Partial financial support under United States Air Force Office of Scientific Research Grant Number 83-0009 to Northwestern University is gratefully acknowledged.

\section{Appendix.-References}

1. Bažant, Z. P., "Crack Band Model for Fracture of Geomaterials," Proceedings of the 4th International Conference on Numerical Methods in Geome- chanics, edited by Z. Eisenstein, Edmonton, Alberta, Canada, Vol. 3, 1982 pp. 1137-1152.

2. Bažant, Z. P., "Distributed Cracking and Nonlocal Continuum," Preprint Europe-U.S. Symposium on Finite Element Methods for Nonlinear Problems, edited by P. Bergan, Norwegian Institute of Technology, Trondheim, Aug. 1985 .

3. Bažant, Z. P., "Fracture in Concrete and Reinforced Concrete," Mechanics of Geomaterials: Rocks, Concrete, Soils, edited by Z. P. Bažant, J. Wiley and Sons, London, England, 1985, pp. 259-303.

4. Bažant, Z. P., "Fracture Mechanics and Strain-Softening in Concrete," Proceedings (U.S.-Japan Workshop, Tokyo, 1985), Finite Element Analysis of Reinforced Concrete Structures, ASCE, C. Meyer and H. Okamura, Eds., pp 121-150.

5. Bažant, Z. P., "Imbricate Continuum and Its Variational Derivation," Journal of Engineering Mechanics, ASCE, Vol. 110, No. 12, Dec., 1984, pp. 1693-1712.

6. Bažant, Z. P., "Mechanics of Distributed Cracking," Applied Mechanics Reviews, Vol. 39, May, 1986, pp. 675-705.

7. Bažant, Z. P., "Mechanics of Fracture and Progressive Cracking in Concrete Structures," Fracture Mechanics Applied to Concrete Structures, edited by G. C. Sih, Martinus Nijhoff Publishers V.B., The Hague, The Netherlands, 1984 pp. 1-94.

8. Bažant, Z. P., and Belytschko, T. B., "Wave Propagation in a Strain-Softening Bar: Exact Solution," Journal of Engineering Mechanics, ASCE, Vol. 111, No. EM3, Mar., 1985, pp. 381-389.

9. Bažant, Z. P., Belytschko, T. B., and Chang, T. P., "Continuum Theory for Strain-Softening," Journal of Engineering Mechanics, ASCE, Vol. 110, No. EM12, Dec., 1984, pp. 1666-1692.

10. Bažant, Z. P., and Cedolin, L., "Fracture Mechanics of Reinforced Concrete," Journal of the Engineering Mechanics Division, ASCE, Vol. 106, No. EM6, Dec., 1980, pp. 1287-1306.

11. Bažant, Z. P., and Cedolin, L., Closure to "Fracture Mechanics of Reinforced Concrete," Journal of the Engineering Mechanics Division, ASCE, Vol. 108, No. EM2, Apr., 1982, pp. 466-471.

12. Bažant, Z. P., and Chang, T. P., "Instability of Nonlocal Continuum and Strain Averaging," Journal of Engineering Mechanics, ASCE, Vol. 110, No. EM10, Oct., 1984, pp. 1441-1450.

13. Bažant, Z. P., and Oh, B. H., "Crack Band Theory for Fracture of Concrete," Matériaux et Constructions (RILEM, Paris) Vol. 16, No. 93, May-June, 1983 pp. 155-177.

14. Bažant, Z. P., and Oh, B. H. "Microplane Model for Progressive Fracture of Concrete and Rock," Journal of Engineering Mechanics, ASCE, Vol. 111, No. EM4, Apr., 1985, pp. 569-582.

15. Belytschko, T., Bažant, Z. P., Hyun, Y. W., and Chang, T. P., "Strain-Softening Materials and Finite Element Solutions," Computers and Structures, Vol 23, No. 2, 1986, pp. 163-180.

16. Crisfield, M. A., "Difficulties with Current Numerical Models for Reinforced Concrete and Some Tentative Solutions," Proceedings, International Conference on "Computer Aided Analysis and Design of Concrete Structures," held in Split, Yugoslavia, Sept. 1984, edited by F. Damjanić, E. Hinton et al., in Split, Yugoslavia, Sept. 1984, edited by F. Damjanić, E.
published by Pineridge Press, Swansea, U.K., pp. 331-358.

17. Darwin, D., and Dodds, R., Discussion of "Fracture Mechanics of Reinforced Concrete," by Z. P. Bažant and L. Cedolin, Journal of the Engineering Mechanics Division, ASCE, Vol. 108, No. EM2, Apr., 1982, pp. 464-466.

18. Dugdale, D. S., "Yielding of Steel Sheets Containing Slits," Journal of the Mechanics and Physics of Solids, Vol. 8, 1960, pp. 100-108.

19. Eringen, A. C., and Ari, N., "Nonlocal Stress Field at Griffith Crack," Cryst. Latt. Def. and Amorph. Mat., Vol. 10, 1983, pp. 33-38.

20. Eringen, A. C., and Edelen, D. G. B., "On Nonlocal Elasticity," International 
Journal of Engineering Science, Vol. 10, 1972, pp. 233-248.

21. Evans, R. H., and Marathe, M. S., "Microcracking and Stress-Strain Curves for Concrete in Tension," Matériaux et Constructions, No. 1, Jan.-Feb., 1968, pp. 61-64.

22. Kröner, E., "Interrelations 'Between Various Branches of Continuum Mechanics," Mechanics of Generalized Continua, edited by E. Kröner, SpringerVerlag, Heidelberg, Germany, 1968, pp. 330-340.

23. Krumhansl, J. A., "Some Considerations of the Relation Between Solid State Physics and Generalized Continuum Mechanics," Mechanics of Generalized Continua, edited by E. Kroner, Springer-Verlag, Heidelberg, Germany, 1968, pp. 298-311.

24. Kunin, I. A., "The Theory of Elastic Media with Microstructure and the The ory of Dislocations," Mechanics of Generalized Continua, edited by E. Kröner Springer-Verlag, Heidelberg, Germany, 1968, pp. 321-328.

25. Levin V. M. "The Relation Between Mathematical Expectations of Stress and Strain Tensors in Elastic Microheterogeneous Media," Journal of Applied Mathematics and Mechanics (translation of Prikladnaya Matematika $i$ Mekhanika), Mathematics and Mechanics (translation
Vol. 35, No. 4, 1971, pp. 694-701.

26. Petersson, P. C., "Crack Growth and Development of Fracture Zones in Plain Concrete and Similar Materials," Doctoral Dissertation, presented to the Lund Institute of Technology, at Lund, Sweden, in 1981, in partial fulfillment of the requirements for the Degree of Doctor of Philosophy.

27. Pietruszczak, S. and Mróz, Z. "Finite Element Analysis of Deformation of Strain-Softening Materials," International Journal of Numerical Methods in Engineering, Vol. 17, 1981, pp. 327-334.

28. Read, H. E., and Hegemier, G. A., "Strain Softening of Rock, Soil and Concrete-A Review Article," Mechanics of Materials, Vol. 3, No. 4, Dec., 1984, pp. 271-294.

29. Sandler, I., and Wright, J., "Summary of Strain-Softening, Theoretical Foundations for Large-Scale Computations of Nonlinear Material Behavior," Preprints: DARPA-NSF Workshop, edited by S. Nemat-Nasser, Northwestern prints: DARPA-NSF Workshop, edited by S. Nemat

30. Valanis, K. C., "On the Uniqueness of Solution to the Initial Value Problems in Softening Materials," ASME Reprint No. 85-APM-29, presented at Joint ASCE-ASME Mechanics Conference, Albuquerque, N.M., June 26, 1985 (to appear in Journal of Applied Mechanics, ASME).

31. Willam, K. I. Bicanic, N., Sture, S., "Constitutive and Computational Aspects of Strain-Softening and Localization in Solids," Proceedings, Sym. on Constitutive Equations, Micro, Macro \& Computational Aspects, edited by K. J. Willam, held at ASME Winter Annual Meeting, New Orleans, Dec., 1984, ASME, New York, N.Y., 1984.

32. Willam, K. J., Hurlbut, B., Sture, S., "Experimental, Constitutive and Computational Aspects of Concrete Failure," Preprints, U.S.-Japan Seminar on Finite Element Analysis of Reinforced Concretes, Tokyo, Japan, May, 1985, pp. 149nite 172 .

33. Wu, F. H., and Freund, L. B., "Deformation Trapping Due to Thermoplastic Instability in One-Dimensional Wave Propagation," Journal of the Mechanics and Physics of Solids, Vol. 32, No. 21, 1984, pp. 119-132.

34. Yuzugullu, O., and Schnobrich, W. C., "A Numerical procedure for the De termination of the Behavior of a Shear Wall Frame System, American Concrete Institute Journal, Vol. 70, July, 1973, pp. 474-479. 